\title{
I feel good whether my friends win or my foes lose: \\ Brain mechanisms underlying feeling similarity
}

\author{
Tatjana Aue \\ Swiss Center for Affective Sciences, University of Geneva, Geneva, Switzerland \\ Center for Cognitive and Social Neuroscience, University of Chicago, IL, USA
}

\author{
Corresponding author: \\ Tatjana Aue \\ Swiss Center for Affective Sciences \\ University of Geneva \\ Biotech Campus \\ Case postale 60 \\ 1211 Geneva 20, Switzerland \\ Phone: +41227415130 \\ e-mail: tatjana.aue@unige.ch
}

Funding:

This work was supported by National Institute of Mental Health Grant No. P50 MH72850. 


\title{
Running head: Feel good
}

\begin{abstract}
People say they enjoy both seeing a preferred social group succeed and seeing an adversary social group fail. At the same time, they state they dislike seeing a preferred social group fail and seeing an adversary social group succeed. The current magnetic resonance imaging study investigated whether-and if so, how—such similarities in reported feeling states are reflected in neural activities. American football fans anticipated success and failure situations for their favorite or their adversary teams. The data support the idea that feeling similarities and divergences expressed in verbal reports carry with them significant neural similarities and differences. Desired (favorite team likely to win and adversary team likely to lose) rather than undesired (favorite team likely to lose and adversary team likely to win) outcomes were associated with heightened activity in the supramarginal gyrus, posterior cingulate cortex, insula, and cerebellum. Precuneus activity additionally distinguished anticipated desirable outcomes for favorite versus adversary teams.
\end{abstract}

Keywords: functional magnetic resonance imaging; social groups; success; failure; desirability 


\title{
I feel good whether my friends win or my foes lose: Brain mechanisms underlying feeling similarity
}

\begin{abstract}
1 Introduction
I support two teams: the All Blacks, and whoever is playing against Australia.
\end{abstract}

This famous slogan among supporters of New Zealand rugby describes a phenomenon that is the rule rather than the exception in sports competitions. Along these lines, soccer fans have been observed to experience Schadenfreude (also called malicious joy or malicious pleasure) when an adversary soccer team loses (Leach, Spears, Branscombe, \& Doosje, 2003). Across a variety of sports settings, the favorite team winning (losing) and the adversary team losing (winning) are said to provoke similar feelings. Evidently, both success and failure can provoke feelings of pleasure or pain, depending on who is experiencing these outcomes. Something positive happening to our beloved can be as delightful as something negative happening to our enemies. ${ }^{1}$

The above-described rugby example refers to social situations that are associated with a similar subjective feeling state, yet differ remarkably with respect to the hedonic valence of the team in question (favorite vs. adversary), as well as with respect to the hedonic valence of the outcome (success vs. failure). Both the favorite team and the fact of winning a game per se have pleasant connotations. Conversely, both the adversary team and the fact of losing a game have unpleasant connotations. The resulting feeling state can then be defined as a combined function 
of team valence and outcome valence. It is thus the situated social meaning of success and failure that makes the experience good or bad.

In the sports context as much as in other social contexts, both the favorite team winning and the adversary team losing are desirable because both can improve the standing of one's own group with respect to rivals and thus serve a similar goal (Taijfel, Flament, Billig, \& Bundy, 1971). The standing of the social group one identifies with is an important component of one's social identity and might significantly contribute to personal self-esteem (Hogg \& Abrams, 1990; J. C. Turner \& Onorato, 1999).

Self-esteem, in turn, has been observed to be an important determinant in the experience of Schadenfreude. Schadenfreude at the failure of another person has been shown to vary as a positive function of self-experienced inferiority or threat to self-evaluation (e.g., van Dijk, Ouwerkerk, Wesseling, \& van Koningsbruggen, 2011; van Dijk, van Koningsbruggen, Ouwerkerk, \& Wesseling, 2011).

In sum, the outcomes of quite different events (e.g., favorite team winning and adversary team losing) lead to strikingly similar feeling states expressed in verbal reports (such as revealed in the rugby citation above). At the same time, these feeling states can be contrasted with those that are supposed to be opposite and relate to undesirable outcomes (i.e., favorite team losing and adversary team winning). The question to be asked here is: Are these similarities and differences in outcome desirability and associated feeling states as revealed in verbal reports merely superficial, or do they carry with them significant neural similarities and differences? And if there are corresponding neural similarities and differences, where are these located?

Numerous studies have investigated the neural correlates of simple stimulus valence (i.e., preference; Anderson et al., 2003; Koeneke, Pedroni, Dieckmann, Bosch, \& Jäncke, 2008; Kringelbach, O’Doherty, Rolls, \& Andrews, 2003) or success and failure (i.e., obtaining a reward 
or winning a game; Kringelbach, 2005; Schoenbaum \& Roesch, 2005; Small et al., 2005; Xue et al., 2009). In general, positive (i.e., personally rewarding) outcomes have been reported to activate the human reward system, with the amygdala, the ventral striatum, and the medial orbitofrontal cortex (mOFC) as key regions (Berridge, Robinson, \& Aldridge, 2009; Grabenhorst \& Rolls, 2009; Hahn et al., 2009; Ursu \& Carter, 2005; Walter, Abler, Ciaramidaro, \& Erk, 2005). Walter et al. (2005), for instance, suggested that the amygdala is implicated in the experience of reward intensity, the ventral striatum in reward prediction and its salience, and the OFC in the processing of reward value. Interestingly with respect to the current research question, the ventral striatum has been linked to Schadenfreude (Takahashi et al., 2009) as well. But are social rewards (and punishments) based on intergroup competitions similarly coded in these areas? Outcomes that increase the standing of a social group that an individual identifies with (e.g., favorite sports team success, adversary sports team failure) might be directly related to increased self-esteem or social status (Hogg \& Abrams, 1990) and therefore be experienced as personally rewarding. In line with this idea, recent research suggests ventral striatal activity to be involved in processing signals of personal power and social status (e.g., Erk, Spitzer, Wunderlich, Galley, \& Walter, 2002). Thus, feeling similarities, such as those stated in the rugby citation, might be expected to be mediated by activity in the human reward system. In addition, there is evidence that an at least partly overlapping network (comprising the amygdala, insula, dorsal striatum, and mOFC) might mediate social cognitive phenomena such as in-group favoritism and out-group negativity (Beer et al., 2008). Consistent with this observation, anticipated social reward, such as the anticipation of a favorite team's soccer goals, has been associated with dorsal striatal activity, notably in the putamen (McLean et al., 2009). Moreover, there is evidence that the putamen is implicated in the experience of Schadenfreude in interindividual interactions (Dvash, Gilam, Ben-Ze'ev, Hendler, \& Shamay-Tsoory, 2010; 
Takahashi et al., 2009). Consequently, regions such as the amygdala, dorsal and ventral striatum, and $\mathrm{mOFC}$ are candidates for the display of social outcome desirability and associated feeling state similarities and differences.

To address these themes, in the current study, the results of prior studies on personal rewards were extended by situating success and failure into a social group context. The neural correlates resulting from feelings of pleasure and pain associated with the anticipation of success and failure of differentially preferred social groups were investigated here by using an eventrelated functional magnetic resonance imaging (fMRI) design. Participants in the current study were American football fans who were given background information suggesting a high or a low likelihood of different National Football League (NFL) teams winning an all-important game. Outcome desirability—associated with a positive feeling state—referred to cases when the favorite team was likely to win or the adversary team was likely to lose. Conversely, outcome undesirability—associated with a negative feeling state—referred to cases when the favorite team was likely to lose or the adversary team was likely to win. Importantly, in each experimental trial, information was given for a single team only (i.e., the other team in the competition remained anonymous), thus permitting the disentangling of effects stemming from the anticipation of favorite team successes versus adversary team failures, as well as effects stemming from the anticipation of favorite team failures versus adversary team successes. In each experimental trial, participants estimated what they thought would be the exact chances of winning for the team in question. $^{2}$

2 Material and methods

\subsection{Participants}


Thirteen (two female) healthy University of Chicago students were recruited via ads posted on the Internet and in university buildings. They were aged between 19 and 34 years $(M=$ $21.8, S D=3.82)$ and indicated elevated interest in American football $(M=5.8, S D=1.63$, on a scale ranging from 1 [no interest] to 7 [intense interest]). All of them were right-handed, had normal or corrected-to-normal vision, and were not currently seeking treatment for affective disorders. Participants were paid $\$ 15 / \mathrm{hr}$.

\subsection{Experimental Design}

A $2 \times 2$ within-subjects design resulted from the manipulation of two factors:

1. Team (favorite and adversary). Before starting with the experiment, from a list depicting all of the 32 NFL teams, participants chose the team they most liked and the team they most disliked. Participants also selected a neutral team, a team they neither liked nor disliked (filler trials, irrelevant to the current research questions).

2. Situation (low and high likelihood of success). The objective likelihood of winning for a given team was classified into two categories: low likelihood of success (LLS; 8, 11, 13, 15, 16, 19, 22, 24, 27, and 30\%) and high likelihood of success (HLS; 68, 71, 74, 76, 79, $82,83,85,87$, and $90 \%$; see Section 2.3 for further details).

\subsection{Procedure}

The study was approved by a local ethics committee (Institutional Review Board for the Biological Sciences at the University of Chicago). Written informed consent was obtained in accordance with the Helsinki Declaration of Human Rights (World Medical Association, 1999). Upon the participants' arrival at the laboratory, the nature of the experiment was explained and informed consent was obtained. Participants then selected their favorite, neutral, and adversary teams. Before beginning the study, the participants had an opportunity to go through 10 practice trials to become familiar with the task. Participants were informed that they could ask questions 
about anything that was not perfectly clear to them. The 40 relevant experimental trials ( 20 for the favorite team, another 20 for the adversary team) were performed while the participants were in an MRI scanner. An additional 20 neutral team trials (concerning teams that the participants did not feel either positive or negative about) constituted filler trials. ${ }^{3}$ For each team, participants estimated probabilities in different situations (10 LLS and 10 HLS trials; see Section 2.2 for details).

In each trial, participants were given background information about a single team (favorite, neutral, or adversary). Participants were explicitly informed that the three teams they selected had, at no time, played against each other and that every experimental trial was independent of what had happened earlier to the same team. Three pieces of background information were presented: (1) the winning odds for the team in question, if a given player played; (2) the same odds, if this specific player did not play; and (3) the odds that this player would actually play. An example of the task is as follows:

(1) The odds that the Chicago Bears will win their first playoff game if their starting quarterback plays are $.84,(2)$ the odds that the Chicago Bears will win their first playoff game if their starting quarterback does not play are .71, and (3) the odds that the Chicago Bears starting quarterback will play are .62. What are the odds that the Chicago Bears win their first playoff game?

The particular probabilities used for the favorite, neutral, and adversary teams were counterbalanced across participants. In order to make the teams more salient, in every trial, the club banners appeared in each of the four corners of the computer screen with the background information presented in-between these banners. The participants' task was to specify what they 
believed to be the odds that the presented team would win its first playoff game, given this information. An example of a trial sequence is shown in Figure 1.

Insert Figure 1 about here

After the study, the participants indicated their affection for the different teams on three different Likert scales. These scales asked how much the participants liked the teams (from 1 [extremely dislike] to 7 [extremely like]), as well as how pleasant (from 1 [extremely unpleasant] to 7 [extremely pleasant]) and appealing (from 1 [extremely unappealing] to 7 [extremely appealing]) the teams were to them.

\subsection{Setting and Apparatus}

MRI data were acquired on a 3T GE Signa scanner (GE Medical Systems, Milwaukee, WI) with a standard quadrature GE head coil. Stimulus presentation and collection of participants' button presses were controlled by e-prime 1.1 (Psychology Software Tools, Inc., Pittsburgh, PA) running on a PC. Participants viewed the statements and club banners by the use of binocular goggles mounted on the head coil approximately 2 inches $(\sim 5 \mathrm{~cm})$ above the participants' eyes. Button press responses were made on two MRI-compatible response boxes (with 0 corresponding to the leftmost button of the left button box and 9 corresponding to the rightmost button of the right button box). Thus, when specifying a subjective likelihood of winning of $54 \%$ for a given team, the participant first pressed the leftmost button of the right button box and subsequently the rightmost button of the left button box.

High-resolution volumetric anatomical images (T1-weighted spoiled gradient-recalled [SPGR] images) were collected for every participant in 1241.5 -mm sagittal slices with a $6^{\circ}$ flip angle and a 24-cm field of view (FOV). Functional data were acquired using a forward/reverse 
spiral acquisition with 40 contiguous $4.2-\mathrm{mm}$ coronal slices with $.5-\mathrm{mm}$ gaps, for an effective slice thickness of $4.7 \mathrm{~mm}$, in an interleaved order spanning the whole brain. Volumes were collected continuously with a repetition time (TR) of $3 \mathrm{~s}$, an echo time (TE) of $28 \mathrm{~ms}$, and an FOV of $24 \mathrm{~cm}$ (flip angle $=84^{\circ}, 64 \times 64$ matrix size, fat suppressed).

\subsection{Data Analysis}

2.5.1 Behavioral data. For each trial, the objectively correct winning odds were subtracted from the participants' estimates, thus yielding 60 difference scores per participant (20 for the neutral filler trials). Missing responses and outliers (estimates referring to difference scores deviating more than three standard deviations from the mean difference score of a participant) made up approximately $5 \%$ of the data and were eliminated. A $2 \times 2$ analysis of variance (ANOVA) with the factors Team (favorite vs. adversary) and Situation (HLS vs. LLS) with an $\alpha$ level of .05 was calculated on these difference scores. A similar ANOVA was conducted on the time needed for response indication (i.e., response time; determined with respect to the onset of the right slide depicted in Figure 1 and based on logarithmic values because of positive skewness. For better visualization and comprehension, Figure 2, described in Section 3.1, displays the initial, untransformed response times).

2.5.2 fMRI data. Functional scans were realigned, normalized, time and motion corrected, temporally smoothed by a low-pass filter consisting of a 3-point Hamming window, and spatially smoothed by a 5-mm full-width at half-maximum (FWHM) Gaussian filter, using AFNI software. Next, a percent signal change data transformation was applied to the functional scans. Individual deconvolution analyses incorporated the generation of impulse response functions (IRFs) of the BOLD signal on a voxel-wise basis (Ward, 2001), permitting the estimation of the hemodynamic response for each condition relative to a baseline state without a priori assumptions about the specific form of an IRF. ${ }^{4}$ Deconvolution analyses comprised separate regressors for 
each time point (six included TRs, starting with the apparition of the club banners and the statement-related probabilities, i.e., the calculation process) of each of the six experimental conditions (favorite HLS, favorite LLS, neutral HLS, neutral LLS, adversary HLS, adversary LLS) and fitted these regressors by the use of a linear least squares model. The estimated individual percent signal change for TRs 2 to 6 was averaged for each voxel in each condition for use in later statistical analyses. Output from the individual deconvolution analyses was converted to Talairach stereotaxic coordinate space (Talairach \& Tournoux, 1988) and interpolated to volumes with $3 \mathrm{~mm}^{3}$ voxels.

Region of interest (ROI) analyses comprising a 2 (favorite vs. adversary team) $\times 2$ (HLS vs. LLS) ANOVA were performed on the observed percent signal changes of each participant for striatal structures (putamen, caudate, nucleus accumbens; areas based on Talairach Daemon database for $A F N I$ ) and the amygdala (Talairach Daemon database), as well as for two clusters in the mOFC (mOFC1: medial mid orbital gyrus, hand drawn based on the MNI coordinate atlas; mOFC2: medial rectal gyrus, hand drawn based on the MNI coordinate atlas; both were subsequently translated into Talairach stereotaxic coordinate space [nonlinear transformation]).

In addition, in a whole-brain analysis, a 2 (favorite vs. adversary team) $\times 2$ (HLS vs. LLS) ANOVA was performed on the observed percent signal changes of each participant. Results were then entered into a cluster analysis with an individual voxel threshold of $p<.01$, minimum cluster connection radius of 5.2, and a cluster volume of $702 \mu \mathrm{L}$ (corresponding to 26 active contiguous voxels). The minimum cluster volume was calculated by a Monte Carlo simulation with 10,000 iterations, assuming some interdependence between voxels (5-mm FWHM), resulting in a corrected whole-brain $p$ value of .05. FMRI data corresponding to missing responses and outliers in the behavioral data (see Section 2.5.1) were excluded from all analyses. 
3 Results

\subsection{Behavioral Data}

The 2 (Team) $\times 2$ (Situation) ANOVA conducted on the participants' estimated winning odds revealed a marginally significant main effect of Team, $F(1,12)=4.52, p<.06, \eta^{2}=.27$. Participants' estimates were larger for the favorite than they were for the adversary team (mean deviations from the objectively correct winning odds: 0.96 and -1.27 , for favorite and adversary, respectively; Figure 2a). Further, there was a significant main effect of Situation, $F(1,12)=$ $11.26, p=.006, \eta^{2}=.48$ (mean deviations from the objectively correct winning odds: .29 and .60 , respectively), signaling, not surprisingly, an overestimation of LLS with regard to HLS probabilities. The interaction term Team $\times$ Situation was not significant, $F(1,12)<1, n s, \eta^{2}=$ .07 .

Insert Figure 2 about here

Regarding the time needed for response indication, the corresponding ANOVA yielded both a significant main effect of Situation, $F(1,12)=6.51, p=.025, \eta^{2}=.35$ (mean response times: $2317 \mathrm{~ms}$ and $2124 \mathrm{~ms}$, for HLS and LLS, respectively), and a significant interaction Team $\times$ Situation, $F(1,12)=9.20, p=.01, \eta^{2}=.43\left(\right.$ main effect of Team: $F(1,12)=0.00, n s, \eta^{2}=.00$; Figure 2b). Post hoc Tukey tests for the interaction of Team and Situation revealed slower response times for adversary team HLS than for adversary team LLS $(p<.015)$, with no difference between the remaining conditions (all $p s>.18$ ). Thus, for the adversary team, participants took more time to specify HLS than LLS, whereas there was no difference between the two situations for the favorite team. 
The favorite team was more liked than the adversary team, and it was judged as more pleasant and more appealing (see Aue et al., 2012, for details). The 13 participants specified nine different favorite teams and eight different adversary teams. Consequently, the results presented herein are not restricted to specific NFL teams.

\section{2 fMRI Data}

\subsubsection{ROI Analyses}

Table 1 displays the results of the 2 (Team) $\times 2$ (Situation) ANOVAs performed for the ROIs. Somewhat surprisingly, none of the ANOVAs yielded any significant effect. A marginally significant interaction Team $\times$ Situation was revealed in the left and right putamen. Pairwise post hoc Tukey tests for the interaction Team $\times$ Situation failed to reach significance for both left and right putamen (all $p \mathrm{~s}>.20$ ). Descriptively, HLS situations were associated with greater activity in the left and right putamen when paired with the favorite team than when paired with the adversary team. For LLS situations, it was the reverse. Because of the high number of ROIs included in this study, I will abstain from overinterpreting the effects in the putamen.

Insert Table 1 about here

\subsubsection{Whole-Brain Analysis}

The 2 (Team) $\times 2$ (Situation) ANOVA revealed a main effect of Team in two areas in the dorsomedial prefrontal cortex (dmPFC; Table 2) and the superior parietal lobule/precuneus. In all cases, favorite team trials were associated with higher activity than were adversary team trials (for more detailed information, see Aue et al., 2012). A main effect of Situation was revealed in the right somatosensory cortex and the left cerebellum. Both areas were more strongly activated for LLS than for HLS situations. 
Insert Table 2 about here

More important to the current research question, the ANOVA yielded a significant interaction of Team and Situation in five regions: the left inferior parietal cortex/supramarginal gyrus (Figure 3a), the right insula (Figure 3b), the right posterior cingulate cortex (PCC; Figure 3c), the bilateral medial precuneus (Figure 3d), and the left cerebellum (Figure 3e; see Table 2 for statistical specifications).

Pairwise post hoc Tukey tests for these significant interactions were subsequently performed. In the inferior parietal cortex, the insula, and the cerebellum, desirable trials (favorite team HLS and adversary team LLS) were characterized by greater activity than were undesirable trials (favorite team LLS and adversary team HLS; specifically, favorite HLS vs. favorite LLS: $p s$ $<.0004, .005$, and .02 , respectively; favorite HLS vs. adversary HLS: $p$ s <.001, .02, and .002; favorite LLS vs. adversary LLS: $p$ s $<.009, .003$, and .007 ; and adversary HLS vs. adversary LLS: $p s<.04, .02$, and .0008). The pattern for the PCC was virtually the same (favorite HLS vs. adversary HLS: $p<.02$; adversary HLS vs. adversary LLS: $p<.007)$, except that the difference in favorite LLS versus adversary LLS only approached significance $(p<.06)$. Similarly, the difference in favorite HLS versus favorite LLS was not significant $(p=.11)$.

It is crucial to note that for each of the four clusters, no difference was observed between the favorite HLS and the adversary LLS (all $p \mathrm{~s}>.16$ ) or between the favorite LLS and the adversary HLS (all $p \mathrm{~s}>.51$ ) conditions. Thus, whereas there were clear neural differences between the events that were expected to provoke different feeling states, there was, at the same time, no difference in activation between events that were supposed to provoke similar feeling states. 
For the precuneus, the observed pattern was different. Here it was the favorite HLS situation that provoked the strongest activity and could be distinguished from all other experimental situations (favorite HLS vs. favorite LLS: $p<.003$; favorite HLS vs. adversary HLS: $p<.0003$; favorite HLS vs. adversary LLS: $p<.03$ ). In contrast to the other clusters, there was no difference between the anticipated failure of the favorite team and the anticipated failure of the adversary team (favorite LLS vs. adversary LLS: $p>.54$ ). In accordance with the other clusters, though, greater activity was observed when the adversary team was likely to lose than when it was likely to win the game $(p<.02)$. Finally, the difference between the trials in which the favorite team was likely to lose and the adversary team was likely to win failed to reach significance (favorite LLS vs. adversary HLS: $p>$.17).

Insert Figure 3 about here

\section{Discussion}

The current study investigated whether similarities in feeling states that are associated with strikingly different events, such as the success of the in-group and the failure of an outgroup, are merely superficial (i.e., appearing only on the level of verbal reports) or whether they carry with them significant neural similarities. Additionally, it examined whether divergences in feeling states are associated with activity differences in the same brain areas. From a literature review on personal rewards and in-group identification and favoritism, it was hypothesized that the human reward system is implicated in the display of similarities and divergences in desirability of social outcomes and associated feeling states. Activity in multiple brain regions was indeed higher when the expected outcome was desirable (favorite team was likely to win and adversary team was likely to lose) than when the expected outcome was undesirable (favorite 
team was likely to lose and adversary team was likely to win), highlighting the fact that the participants experienced success and failure differently as a function of social group. ${ }^{5}$

Importantly, though, the most prominent areas of the human reward system (cf. Berridge et al., 2009; Grabenhorst \& Rolls, 2009; Hahn et al., 2009; Ursu \& Carter, 2005; Walter et al., 2005) that are typically activated in personal reward situations - the amygdala, the ventral striatum, and the mOFC — were not among the regions that displayed outcome desirability in the current study. Only the PCC and the dorsal striatum (putamen; marginal effect) distinguished between desirable and undesirable social outcomes, two regions that, in a broader sense, can also be considered substituents of the human reward system. Because of the high number of ROIs included in this study and the nonsignificance of the effect, however, it is necessary to be prudent in not overinterpreting the findings in the putamen.

Yet, it is noteworthy that dorsal striatal activity has been observed in highly related areas of research. For instance, elevated activity in the dorsal striatum (caudate) has been linked with anticipatory satisfaction during the application of punishment to non-cooperative interaction partners and thus to adversary others (e.g., de Quervain et al., 2004). In addition, this activity is associated with a phenomenon called self-serving bias (Blackwood et al., 2003). Likewise, increased activity in the putamen was revealed in social reward settings (i.e., when participants anticipated a favorite team's soccer goals; McLean et al., 2009) and also during Schadenfreude (Takahashi et al., 2009). That desirability effects in the current study failed to reach significance in the putamen might be related to the comparably low number of participants $(n=13)$ and the limited number of experimental trials per condition $(n=10)$.

PCC activity has been associated with, among other things, the subjective value of monetary rewards (Fujiwara et al., 2009; Kable \& Glimcher, 2007), as well as with pleasant social events such as the experience of being liked (Davey, Allen, Harrison, Dwyer, \& Yucel, 
2010) and partner choice (Funayama et al., 2012). That the present social task did not activate other key structures of the human reward system may suggest that directly self-relevant events are experienced as somewhat different from those resulting from social identification. Yet, given the close association of social events and personal self-esteem (e.g., Hogg \& Abrams, 1990), it is generally difficult to make a clear distinction between personal and social relevance. It is possible that resemblance of personal self-relevance and significant other-relevance varies as a function of distance towards the other person or group — or with the abstractness of the other person or group.

There are, however, several alternative explanations for the lack of finding significant activation in the $\mathrm{mOFC}$, the ventral striatum, and the amygdala. That there was no significant mOFC activation in the supposedly socially rewarding experimental conditions may lie in the fact that participants in the current study did not need to take any action or decision. Recent research suggests this area to be strongly implicated in decision-making contexts and to guide personal actions (Coricelli et al., 2005; Hughes \& Beer, 2012; Nachev, 2006; Noonan, Sallet, Rudebeck, Buckley, \& Rushworth, 2010; Rolls \& Grabenhorst, 2008). In a similar vein, ventral striatum activity might signal reward only when individuals are able to choose between different outcomes (Ursu \& Carter, 2005). Consistent with such an interpretation is that activity in the ventral striatum was found to predict reward value when money could be gained by performing specific actions but not when it was gained passively (Zink, Pagnoni, Martin, Dhamala, \& Berns, 2003).

That the amygdala did not distinguish between desirable and undesirable anticipated outcomes in the current study may be explained by the finding that the amygdala is involved in positive (e.g., Breiter et al., 1996; Hamann \& Mao, 2002) as well as negative affect (e.g., Adolphs et al., 1995; Breiter et al., 1996; LeDoux, 2000; Öhman \& Mineka, 2001). It is thus possible that the amygdala codes personal salience in general (Davis \& Whalen, 2001; Kawashima et al., 1999; Wager, Phan, Liberzon, \& Taylor, 2003; Whalen et al., 2004). The 
anticipation of the adversary team winning or the favorite team losing was possibly experienced as being equally salient to the anticipation of the favorite team winning and the adversary team losing, hence preventing differential amygdala activity.

Instead of the amygdala, a number of additional regions distinguished between desirable and undesirable outcomes in this study, namely, the supramarginal gyrus, the insula, the precuneus, and the cerebellum. All of these areas have been related to positive (or lack of negative) affect in earlier research. Increased activity in the supramarginal gyrus was observed for diverse phenomena such as taste perception in the fasting state (Gautier et al., 1999), perception of happy faces (Phillips et al., 1998), and decisions on a favorite brand (Deppe, Schwindt, Kugel, Plassmann, \& Kenning, 2005); decreased activation in this area was reported during worry (Hoehn-Saric, Lee, McLeod, \& Wong, 2005). Cerebellar lesions reduce the experience of pleasant experience (e.g., B. M. Turner et al., 2007). Both the precuneus and the insula have been related to Schadenfreude when the failures applied to others who were envied (Cikara \& Fiske, 2011; Dvash et al., 2010). Consistent with this observation, the insula has further been associated with in-group favoritism and out-group negativity in the implicit association task (Beer et al., 2008).

That insular activity is often reported to code stimulus unpleasantness (e.g., expectancy of pain) rather than pleasantness (e.g., Grabenhorst \& Rolls, 2009; Ploghaus, Beceraa, Borras, \& Borsook, 2003) should not be surprising. The function of the insula has been suggested to be the mediation of subjective feeling states (cf., Damasio et al., 2000; Phan, Wager, Taylor, \& Liberzon, 2002), and different, but closely located, neural assemblies in the insula may be implicated in negative and positive affect (see Lamm \& Singer, 2010, for details).

Some brain structures that distinguished between desirable and undesirable outcomes in the current study - the inferior parietal lobe (including the supramarginal gyrus), the precuneus, 
and the PCC - are typically activated in perspective-taking or theory-of-mind tasks (e.g., Malhi et al., 2008; Ruby \& Decety, 2004; Vogeley et al., 2004) and thus during the application of other people's experiences to the self. Likewise, the precuneus and the cerebellum have been associated with reflective self-awareness (e.g., Kjaer, Nowak, \& Lou, 2002) and self-regulation (Cavanna \& Trimble, 2006), and the PCC is implicated in highly akin phenomena such as selfknowledge (e.g., Ochsner et al., 2005) and self-relevance (Schmitz \& Johnson, 2007).

The present results extend these findings in that they suggest that these regions are not merely implicated in the processing of self-related information per se, but that they may additionally be sensitive to the desirability of the presented information. Clearly, in the current study, the situations that, based on social identification processes, enhanced self-esteem (i.e., improved the standing of the individual's own football team with respect to rivals) elicited greater activation than those that threatened self-esteem.

It is possible that individuals actively work to increase the impact of self-serving information, for instance, by paying greater attention or applying greater salience to supportive information (see Hahn, Ross, \& Stein, 2006; Schweizer, Alexander, Cusimano, \& Stuss, 2007; Sturm et al., 2006, for the implication of the inferior parietal lobule, the precuneus, the insula, and the cerebellum in attentional processes). An exemplary study by Schiller, Freeman, Mitchell, Uleman, and Phelps (2009) revealed the importance of the PCC for interindividual impression formation. Participants in this study looked at human faces and received several pieces of positive and negative person-descriptive information about each of these faces. Interestingly, the more the PCC was recruited during the encoding of a specific piece of information, the more its valence influenced the participant's overall impression of the person displayed (like vs. dislike). Consistent with this study's results, a contemporary view in attention research sees the PCC as 
important for top-down goal-directed selection of stimuli and responses (e.g., Corbetta \& Shulman, 2002). ${ }^{6}$

Applied to the present study's findings, which demonstrate greater PCC activity for desirable than for undesirable outcomes, it can be hypothesized that in social group situations, humans may have the tendency to apply less salience to undesirable evidence and thereby intensify satisfying desirable experiences. ${ }^{7}$ This may be achieved by paying particularly strong attention to desirable evidence (see Aue et al., 2012, for related issues). Hence, self-serving impressions may be actively enhanced. Intriguingly, all clusters for which there was an effect of desirability revealed greater neural activity with the desirable than with the undesirable evidence. This is consistent with the interpretation of the study results outlined above. If it really were the case that greater salience (i.e., attention) is applied to the desirable evidence in order to augment self-esteem, overall neural activity in attention-related brain regions would be expected to be more enhanced for anticipated desirable outcomes.

Moreover, the observed precuneus activation (highest activation for favorite HLS) suggests that it might be the favorite HLS condition, which is most strongly attended to (for the implication of the precuneus in attention and self-relevance, see Cavanna \& Trimble, 2006) because, overall, this specific experimental condition is most strongly distinguished from the others. From a psychological perspective, this is reasonable because, by the preferential treatment of the own social group, individuals can directly improve their social identity (Tajfel et al., 1971; J. C. Turner \& Onorato, 1999). Treating the out-group unfavorably, by contrast, serves this goal only indirectly. Thus, there may still be a little difference in seeing the favorite team winning versus the adversary team losing. Whether this extends to the associated feeling states remains an open question. 
Further aspects of this study merit particular mention. First, in contrast to numerous earlier studies, an ANOVA was used for the examination of the data. This approach has the advantage of helping to split apart effects from different variance sources and simultaneously controls for alpha inflation, thus facilitating the interpretation of activation patterns (e.g., main effects for Team or Situation in the present study could not be explained by higher order interactions).

Second, verbal reports of feeling state were intentionally not assessed after each trial in order to avoid the establishment of demand characteristics. Instead, the participants were asked to make predictions on the likelihood that a team would win a playoff game. Thus, the observed similarities in neural activations in the current study are not likely to be a result of participants trying to construe feeling states according to predefined social rules. While such a strategy will have reduced the apparition of demand characteristics during the experimental task, not including a postexperimental assessment of the subjective feeling states associated with each experimental condition can be considered a limitation. However, in accordance with the study hypotheses regarding the subjective feeling states, inspection of the response times (Figure 2b) suggests that participants may indeed have had a harder time specifying the winning odds for the adversary team, when the objective likelihood of winning was high rather than low. Such an effect would be consistent with the idea that the participants were at greater ease or more willing (i.e., enjoyed it more) to attribute LLS rather than HLS to the adversary team.

Other limitations of the study that warrant mention include the circumscribed number of participants and the circumscribed number of trials per experimental condition. Increasing both in future experiments could yield additional differences in the areas reported herein to demonstrate neural similarities of supposedly similar feeling states. Nonetheless, there was no general 
problem of statistical power, as demonstrated by the fact that there were indeed differences between desirable and undesirable conditions (i.e., between supposedly different feeling states).

Finally, it is possible that the neural responses regarding the processing of team information (pictorial + verbal presentation) and that of situation information (verbal presentation) act on different time scales, with team information being processed more quickly than situation information. Such differential timing could bias against finding an interaction of the type predicted in the present study. This latter consideration could explain the missing effects in alternative areas typically activated in reward situations - the amygdala, the ventral striatum, and the mOFC. 


\section{References}

Adolphs, R., Tranel, D., Damasio, H., \& Damasio, A. R. (1995). Fear and the human amygdala. Journal of Neuroscience, 15, 5879-5891.

Anderson, A. K., Christoff, K., Stappen, I. Panitz, D., Ghahremani, D. G, Glover, G., ... Sobel, N. (2003). Dissociated neural representations of intensity and valence in human olfaction. Nature Neuroscience, 6, 196-202.

Aue, T., Nusbaum, H. C., \& Cacioppo, J. T. (2012). Neural correlates of wishful thinking. Social Cognitive and Affective Neuroscience, 7, 991-1000.

Bechara, A., Damasio, H., \& Damasio, A. R. (2000). Emotion, decision making and the orbitofrontal cortex. Cerebral Cortex, 10, 295-307.

Beer, J. S., Stallen, M., Lombardo, M. V., Gonsalkorale, K., Cunningham, W. A., \& Sherman, J. W. (2008). The quadruple process model approach to examining the neural underpinnings of prejudice. Neuroimage, 43, 775-783.

Berridge, K. C., Robinson, T. E., \& Aldridge, J. W. (2009). Dissecting components of reward: 'Liking', 'wanting', and learning. Current Opinion in Pharmacology, 9, 65-73.

Blackwood, N. J., Bentall, R. P., ffytche, D. H., Simmons, A., Murray, R. M., \& Howard, R. J. (2003). Self-responsibility and the self-serving bias: An fMRI investigation of causal attributions. Neuroimage, 20, 1076-1085.

Breiter, H. C., Etcoff, N. L., Whalen, P. J., Kennedy, W. A., Rauch, S. L., Buckner, R. L., . . . Rosen, B. R. (1996). Response and habituation of the human amygdala during visual processing of facial expression. Neuron, 17, 875-887.

Cavanna, A. E., \& Trimble, M. R. (2006). The precuneus: A review of its functional anatomy and behavioural correlates. Brain, 129, 564-583. 
Cikara, M., \& Fiske, S. T. (2011). Bounded empathy: Neural responses to outgroup targets' (mis)fortunes. Journal of Cognitive Neuroscience, 23, 3791-3803.

Corbetta, M., \& Shulman, G. L. (2002). Control of goal-directed and stimulus-driven attention in the brain. Nature Reviews Neuroscience, 3, 201-215.

Coricelli, G., Critchley, H. D., Joffily, M., O’Doherty, J. P., Sirigu, A., \& Dolan, R. J. (2005). Regret and avoidance: A neuroimaging study of choice behavior. Nature Neuroscience, 8 , $1255-1262$.

Damasio, A. R. (1994). Descartes' error: Emotion, reason, and the human brain. New York, NY: Grosset/Putnam.

Damasio, A. R., Grabowski, T. J., Bechara, A., Damasio, H., Ponto, L. L., Parvizi, J., \& Hichwa, R. D. (2000). Subcortical and cortical brain activity during the feeling of self-generated emotions. Nature Neuroscience, 3, 1049-1056.

Davey, C. G., Allen, N. B., Harrison, B. J., Dwyer, D. B., \& Yucel, M. (2010). Being liked activates primary reward and midline self-related brain regions. Human Brain Mapping, 31, $660-668$.

Davis, M., \& Whalen, P. J. (2001). The amygdala: Vigilance and emotion. Molecular Psychiatry, 6, 13-34.

Deppe, M., Schwindt, W., Kugel, H., Plassmann, H., \& Kenning, P. (2005). Nonlinear responses within the medial prefrontal cortex reveal when specific implicit information influences economic decision making. Journal of Neuroimaging, 15, 171-182.

de Quervain, D. J., Fischbacher, U., Treyer, V., Schellhammer, M., Schnyder, U., Buck, A., \& Fehr, E. (2004). The neural basis of altruistic punishment. Science, 305, 1254-1258.

Dvash, J., Gilam, G., Ben-Ze'ev, A., Hendler, T., \& Shamay-Tsoory, S. G. (2010). The envious brain: The neural basis of social comparison. Human Brain Mapping, 31, 1741-1750. 
Erk, S., Spitzer, M., Wunderlich, A. P., Galley, L., \& Walter, H. (2002). Cultural objects modulate reward circuitry. Neuroreport, 13, 2499-2503.

Fujiwara, J., Tobler, P. N., Taira, M., Iijima, T., \& Tsutsui, K. I. (2009). Segregated and integrated coding of reward and punishment in the cingulate cortex. Journal of Neurophysiology, 101, 3284-3293.

Funayama, R., Sugiura, M., Sassa, Y., Jeong, H., Wakusawa, K., Horie, K., . . Kawashima, R. (2012). Neural bases of human mate choice: Multiple value dimensions, sex difference, and self-assessment system. Social Neuroscience, 7, 59-73.

Gautier, J. F., Chen, K. W., Uecker, A., Bandy, D., Frost, J., Salbe, A. D., . . Tataranni, P. A. (1999). Regions of the human brain affected during a liquid-meal taste perception in the fasting state: A positron emission tomography study. American Journal of Clinical Nutrition, 70, 806-810.

Gazzola, V., \& Keysers, C. (2009). The observation and execution of actions share motor and somatosensory voxels in all tested subjects: Single-subject analyses of unsmoothed fMRI data. Cerebral Cortex, 19, 1239-1255.

Grabenhorst, F., \& Rolls, E. T. (2009). Different representations of relative and absolute subjective value in the human brain. Neuroimage, 48, 258-268.

Hahn, T., Dresler, T., Ehlis, A. C., Plichta, M. M., Heinzel, S., Polak, T., . . Fallgatter, A. J. (2009). Neural response to reward anticipation is modulated by Gray's impulsivity. Neuroimage, 46, 1148-1153.

Hahn, B., Ross, T. J., \& Stein, E. A. (2006). Neuroanatomical dissociation between bottom-up and top-down processes of visuospatial selective attention. Neuroimage, 32, 842-853.

Hamann, S., \& Mao, H. (2002). Positive and negative emotional verbal stimuli elicit activity in the left amygdala. Neuroreport, 13, 15-19. 
Hoehn-Saric, R., Lee, J. S., McLeod, D. R., \& Wong, D. F. (2005). Effect of worry on regional cerebral blood flow in nonanxious subjects. Psychiatry Research: Neuroimaging, 140, 259269.

Hogg, M. A., \& Abrams, D. (1990). Social motivation, self-esteem, and social identity. In D. Abrams \& M. A. Hogg (Eds.), Social identity theory: Constructive and critical advances (pp. 28-47). New York, NY: Springer.

Hughes, B. L., \& Beer, J. S. (2012). Medial orbitofrontal cortex is associated with shifting decision thresholds in self-serving cognition. Neuroimage, 61, 889-898.

Kable, J. W., \& Glimcher, P. W. (2007). The neural correlates of subjective value during intertemporal choice. Nature Neuroscience, 10, 1625-1633.

Kahnt, T., \& Tobler, P. N. (2013). Salience signals in the right temporoparietal junction facilitate value-based decisions. Journal of Neuroscience, 33, 863-869.

Kawashima, R., Sugiura M., Kato, T., Nakamura, A., Hatano, K., Ito, K., . . Nakamura, K. (1999). The human amygdala plays an important role in gaze monitoring: A PET study. Brain, 122, 779-783.

Kjaer, T. W., Nowak, M., \& Lou, H. C. (2002). Reflective self-awareness and conscious states: PET evidence for a common midline parietofrontal core. Neuroimage, 17, 1080-1086.

Koeneke, S., Pedroni, A. F., Dieckmann, A., Bosch, V., \& Jäncke, L. (2008). Individual preferences modulate incentive values: Evidence from functional MRI. Behavioral and Brain Functions, 4, 55.

Kringelbach, M. L. (2005). The human orbitofrontal cortex: Linking reward to hedonic experience. Nature Reviews Neuroscience, 6, 691-702.

Kringelbach, M. L., O’Doherty, J., Rolls, E. T., \& Andrews, C. (2003). Activation of the human orbitofrontal cortex to a liquid food stimulus is correlated with its subjective pleasantness. 
Cerebral Cortex, 13, 1064-1071.

Lamm, C., \& Singer, T. (2010). The role of anterior insular cortex in social emotions. Brain Structure and Function, 214, 579-591.

Leach, C. W., Spears, R., Branscombe, N. R., \& Doosje, B. (2003). Malicious pleasure: Schadenfreude at the suffering of another group. Journal of Personality and Social Psychology, 84, 932-943.

LeDoux, J. E. (2000). Emotion circuits in the brain. Annual Review of Neuroscience, 23, 155184.

Macuga, K. L., \& Frey, S. H. (2012). Neural representations involved in observed, imagined, and imitated actions are dissociable and hierarchically organized. Neuroimage, 59, 2798-2807.

Malhi, G. S., Lagopoulos, J., Das, P., Moss, K., Berk, M., \& Coulston, C. M. (2008). A functional MRI study of theory of mind in euthymic bipolar disorder patients. Bipolar Disorders, 10, 943-956.

McLean, Brennan, D., Wyper, D., Condon, B., Hadley, D., \& Cavanagh, J. (2009). Localisation of regions of intense pleasure response evoked by soccer goals. Psychiatry Research: Neuroimaging, 171, 33-43.

Mellers, B. A. (2000). Choice and the relative pleasure of consequences. Psychological Bulletin, $126,910-924$.

Nachev, P. (2006). Cognition and medial frontal cortex in health and disease. Current Opinion in Neurology, 19, 586-592.

Noonan, M. P., Sallet, J., Rudebeck, P. H., Buckley, M. J., \& Rushworth, M. F. (2010). Does the medial orbitofrontal cortex have a role in social valuation? European Journal of Neuroscience, 31, 2341-2351. 
Ochsner, K. N., Beer, J. S., Robertson, E. A., Cooper, J., Gabrieli, J. D. E., Kihlstrom, J. F., \& D’Esposito, M. (2005). The neural correlates of direct and reflected self-knowledge. Neuroimage, 28, 797-814.

Öhman, A., \&, Mineka, S. (2001). Fears, phobias, and preparedness: Toward an evolved module of fear and fear learning. Psychological Review, 108, 483-522.

Phan, K. L, Wager, T., Taylor, S. F., \& Liberzon, I. (2002). Functional neuroanatomy of emotion: A meta-analysis of emotion activation studies in PET and fMRI. Neuroimage, 16, 331-348.

Phillips, M. L., Bullmore, E. T., Howard, R., Woodruff, P. W. R., Wright, I. C., Williams, S. C. R., . . David, A. S. (1998). Investigation of facial recognition memory and happy and sad facial expression perception: An fMRI study. Psychiatry Research: Neuroimaging, 83, $127-138$.

Ploghaus, A., Beceraa, L., Borras, C., \& Borsook, D. (2003). Neural circuitry underlying pain modulation: Expectation, hypnosis, placebo. Trends in Cognitive Sciences, 7, 197-200.

Rolls, E. T., \& Grabenhorst, F. (2008). The orbitofrontal cortex and beyond: From affect to decision-making. Progress in Neurobiology, 86, 216-244.

Ruby, P., \& Decety, J. (2004). How would you feel versus how do you think she would feel? A neuroimaging study of perspective-taking with social emotions. Journal of Cognitive Neuroscience, 16, 988-999.

Schiller, D., Freeman, J. B., Mitchell, J. P., Uleman, J. S., \& Phelps, E. A. (2009). A neural mechanism of first impressions. Nature Neuroscience, 12, 508-514.

Schmitz, T. W., \& Johnson, S. C. (2007). Relevance to self: A brief review and framework of neural systems underlying appraisal. Neuroscience and Biobehavioral Reviews, 31, 585596.

Schoenbaum, G., \& Roesch, M. R. (2005). Orbitofrontal cortex, associative learning, and 
expectancies. Neuron, 47, 633-636.

Schweizer, T. A., Alexander, M. P., Cusimano, M., \& Stuss, D. T. (2007). Fast and efficient visuotemporal attention requires the cerebellum. Neuropsychologia, 45, 3068-3074.

Small, D. M., Gittelman, D., Simmons, K., Bloise, S. M., Parrish, T., \& Mesulam, M. M. (2005). Monetary incentives enhance processing in brain regions mediating top-down control of attention. Cerebral Cortex, 15, 1855-1865.

Sturm, W., Schmenk, B., Fimm, B., Specht, K., Weis, S., Thron, A., \& Willmes, K. (2006). Spatial attention: More than intrinsic alerting? Experimental Brain Research, 171, 16-25.

Tajfel, H., Flament, C., Billig, M. G., \& Bundy, R. F. (1971). Social categorization and intergroup behaviour. European Journal of Social Psychology, 1, 149-177.

Takahashi, H., Kato, M., Matsuura, M., Mobbs, D., Shara, T., \& Okubo, Y. (2009). When your gain is my pain and your pain is my gain: Neural correlates of envy and Schadenfreude. Science, 323, 937-939.

Talairach, J., \& Tournoux, P. (1988). Co-planar stereotaxic atlas of the human brain: $3 D$ proportional system: An approach to cerebral imaging. New York, NY: Georg Thieme Verlag.

Turner, B. M., Paradiso, S., Marvel, C. L., Pierson, R., Boles Ponto, L. L., Hichwa, R. D., \& Robinson, R. G. (2007). The cerebellum and emotional experience. Neuropsychologia, 45, $1331-1341$.

Turner, J. C., \& Onorato, R. S. (1999). Social identity, personality, and the self concept: A self categorization perspective. In T. R. Tyler, R. M. Kramer, \& O. P. John (Eds.), The psychology of the social self (pp. 11-46). Mahwah, NJ: Erlbaum. 
Ursu, S., \& Carter, C. S. (2005). Outcome representations, counterfactual comparisons and the human orbitofrontal cortex: Implications for neuroimaging studies of decision-making. Cognitive Brain Research, 23, 51-60.

van Dijk, W. W., Ouwerkerk, J. W., Wesseling, Y. M., \& van Koningsbruggen, G. M. (2011). Towards understanding pleasure at misfortunes of others: The impact of self-evaluation threat on Schadenfreude. Cognition and Emotion, 25, 360-368.

van Dijk, W. W., van Koningsbruggen, G. M., Ouwerkerk, J. W., \& Wesseling, Y. M. (2011). Self-esteem, self-affirmation, and Schadenfreude. Emotion, 11, 1445-1449.

Vogeley, K., May, M., Ritzl, A., Falkai, P., Zilles, K., \& Fink, G. R. (2004). Neural correlates of first-person perspective as one constituent of human self-consciousness. Journal of Cognitive Neuroscience, 16, 817-827.

Wager, T., Phan, K. L., Liberzon, I., \& Taylor, S. F. (2003).Valence, gender, and lateralization of functional brain anatomy in emotion: A meta-analysis of findings from neuroimaging. Neuroimage, 19, 513-531.

Walter, H., Abler, B., Ciaramidaro, A., \& Erk, S. (2005). Motivating forces of human actions: Neuroimaging reward and social interaction. Brain Research Bulletin, 67, 368-381.

Ward, B. D. (2001). Deconvolution analysis of fMRI time series data (technical report). Milwaukee: Biophysics Research Institute, Medical College of Wisconsin.

Whalen, P. J., Kagan, J., Cook, R. G., Davis, F. C., Kim, H., Polis, S., . . Johnstone, T. (2004). Human amygdala responsivity to masked fearful eye whites. Science, 306, 2061.

World Medical Association. (1999). Proposed revision of the Declaration of Helsinki. Bulletin of Medical Ethics, 147, 18-22. 
Xue, G., Lu, Z., Levin, I. P., Weller, J. A., Li, X., \& Bechara, A. (2009). Functional dissociations of risk and reward processing in the medial prefrontal cortex. Cerebral Cortex, 19, 10191027.

Zink, F. G., Pagnoni, G., Martin, M. E., Dhamala, M., \& Berns, G. S. (2003). Human striatal response to salient non-rewarding stimuli. Journal of Neuroscience, 23, 8092-8097. 


\section{Footnotes}

${ }^{1}$ Throughout this article, I make no strong distinction between actual outcomes and anticipation of those outcomes (e.g., Schadenfreude vs. anticipated Schadenfreude) because a close correspondence is assumed between (the neural signatures of) feeling states related to anticipated outcomes and those related to real outcomes. First, the literature has revealed that people are capable of using their anticipated feeling states related to imagined outcomes for efficient decision making and that the circuits underlying the mediation of anticipated feeling states and associated decisions rely on the recall (i.e., the reactivation) of earlier real experiences (e.g., somatic marker hypothesis; Bechara, Damasio, \& Damasio, 2000; Damasio, 1994). Second, it has been shown for laboratory experiments that there is a close correspondence between anticipated pleasure and actual pleasure in response to imagined and real outcomes, respectively (e.g., Mellers, 2000).

${ }^{2}$ Notably, the data presented here have also been analyzed with respect to an essentially different research question (neural correlates of biased expectancies [i.e., wishful thinking]; see Aue, Nusbaum, \& Cacioppo, 2012, for details). The focus of this earlier publication is on general social group differences and how group-related differences reflected in neural connectivities relate to the assessed individual biasing of expectancies. The present study instead examines how different "objective" experimental conditions (high likelihood of losing vs. high likelihood of winning) interact with social group information-without any notion of individual biasing of subjective expectancies. Thus, the focus of the current publication is on the feeling states that are related to anticipating favorite and adversary football teams in desirable versus undesirable situations. These feeling states do not need to be related to the biasing of subjective expectancies at all. Consequently, the topics treated in the separate papers are not redundant; rather, they complement each other. 
${ }^{3}$ Whereas postexperimental responses to the questions on how much the participants liked the different teams and how pleasant and appealing these teams were to them suggested that the neutral team would fall exactly in-between the favorite and the adversary team, data acquired during the task (e.g., estimated winning odds, response times) were characterized by virtually identical responses toward the neutral team and the adversary team. Despite the neutral team not having an explicitly negative connotation, the fact that, in everyday life, it happens to be in competition with the favorite team possibly gives it an adversary role, a role that may be activated in competitive social situations. Therefore, the adversary team and the supposedly neutral team may have been treated in a highly similar fashion in the current study. Because of the unclear affective status of the neutral team, no data are reported for it.

${ }^{4}$ For several important reasons, it was decided to model the data with impulse response functions instead of with the standard canonical response function. First, it was desirable to retain comparability with the findings reported in another article. Although that article addressed a different research question (wishful thinking-related neural activity; Aue et al., 2012; cf. Footnote 2), it was based on the same data. Second, given that little is known about the exact cognitive processes and the exact timing underlying the feeling states investigated herein, it seems prudent to not make any assumptions about the specific form of the hemodynamic response. Therefore, the design applied should be characterized by greater flexibility.

Third, adding different events to the statistical design (e.g., corresponding to the different slides depicted in Figure 1) could have hidden the Team $\times$ Situation interactions that are actually present because some participants may have completed the calculation process in the logo and background information period (i.e., team and information recognition + calculation phase;

Figure 1, middle slide), whereas others arrived at a result only later, namely, when a response had to be given (i.e., calculation + response indication phase; Figure 1, right slide). In addition, the 
corresponding feeling states of interest cannot be limited to only one of these experimental phases.

${ }^{5}$ None of the ROIs investigated showed a main effect of Team or Situation. The wholebrain analyses revealed that three areas differentiated between the favorite and the adversary team (two in the dmPFC and one in the superior parietal lobule). These team-specific activations can be explained by selective attention to and differential self-relevance of the described teams (see Aue et al., 2012, for details).

Furthermore, two areas distinguished between LLS and HLS situations (somatosensory cortex and cerebellum). Both regions have been related to motor activity in earlier research (e.g., Gazzola \& Keysers, 2009; Macuga \& Frey, 2012). Given the existence of crossed cerebrocerebellar connections, it is not surprising that the difference for the postcentral gyrus is on the right side and the one in the cerebellum on the left side. Because the LLS and the HLS trials were characterized by differential use of the button boxes (more left-hand movements for LLS situations because of greater use of left button box), it can be hypothesized that motor actions are at the basis of these activations. In order to investigate this hypothesis, additional analyses have been performed to determine whether these differential activities were indeed related to motor actions or instead to cognitions of losing versus winning.

If activity in the right postcentral gyrus and the left culmen indeed originated from differential use of the right and left button boxes, the participants' neural activity in these regions would be expected to increase the more they used the left button box. Therefore, each participant's mean activity in the two clusters (across all experimental conditions) was first extracted. In a second step, the extent of the use of the left button box by each participant was determined. This was done by relating the number of left button presses to the overall number of button presses. Finally, between-subjects Pearson product-moment correlation coefficients 
between mean neural activity in the two clusters and the extent of left button box use were calculated. Consistent with the above-stated hypothesis, the use of the left button box was positively related to neural activities in both the right postcentral gyrus, $r(13)=.43, p=.07$, and the left culmen, $r(13)=.50, p<.05$, both one-tailed.

If, instead, activities in these areas had been related to cognitions of losing versus winning, mean neural activity in these areas would have been expected to vary as a negative function of average indicated likelihood of winning in the behavioral ratings. However, betweensubjects Pearson correlations revealed that average likelihood of winning was unrelated to neural activities in the postcentral gyrus, $r(13)=.06, n s$, and in the culmen, $r(13)=-.03, n s$.

${ }^{6}$ But note that, in the current study, there was an overall decrease in activation in the PCC.

${ }^{7}$ Future research on such salience-related aspects may benefit from a formal definition and behavioral measure of salience (e.g., Kahnt \& Tobler, 2013). 


\section{Figure captions}

Figure 1. Trial sequence. First, the three statements were presented for a random time of 3, 6, or $9 \mathrm{~s}$. At that point, there was no indication of the corresponding probabilities and the team in question. In a next step, the club banner of the team appeared in the four corners of the screen and the statement-related probabilities were projected to the right of the statements. Participants had 9 s to calculate the supposed likelihood of winning. After this time, a question prompted them to specify their estimate of the likelihood of winning by the use of two button boxes. They were given $6 \mathrm{~s}$ to do so. The next trial began immediately afterward, with the presentation of the three statements.

Figure 2. Deviation in estimated winning odds from the objective likelihood of winning (a) and time needed for estimate indication (response time, b). Error bars depict standard errors.

Figure 3: Regions displaying activity modulated by an interaction of Team and Situation. Wholebrain analysis. Error bars depict standard errors. 
Table 1: ROI analyses

\begin{tabular}{|c|c|c|c|c|c|c|c|c|c|}
\hline \multirow[b]{3}{*}{ Region } & \multicolumn{9}{|c|}{ Effects } \\
\hline & \multicolumn{3}{|c|}{ Team } & \multicolumn{3}{|c|}{ Situation } & \multicolumn{3}{|c|}{$\begin{array}{c}\text { Team } \times \\
\text { Situation } \\
\end{array}$} \\
\hline & $F(1,12)$ & $p$ & $\eta^{2}$ & $F(1,12)$ & $p$ & $\eta^{2}$ & $F(1,12)$ & $p$ & $\eta^{2}$ \\
\hline L Amygdala & 0.27 & ns & .02 & 0.24 & $n s$ & .02 & 2.51 & $n s$ & .17 \\
\hline R Amygdala & 0.03 & $n s$ & .00 & 0.40 & $n s$ & .03 & 1.76 & $n s$ & .13 \\
\hline R Caudate & 0.07 & ns & .01 & 0.80 & $n s$ & .06 & 0.06 & $n s$ & .01 \\
\hline L Nucleus accumbens & 0.00 & $n s$ & .00 & 0.35 & $n s$ & .03 & 1.04 & $n s$ & .08 \\
\hline R Nucleus accumbens & 0.02 & $n s$ & .00 & 0.87 & $n s$ & .07 & 0.13 & $n s$ & .01 \\
\hline OFC1: Medial Mid Orbital Gyrus & 2.58 & $n s$ & .18 & 1.25 & $n s$ & .09 & 2.82 & $n s$ & .19 \\
\hline OFC2: Medial Rectal Gyrus & 0.01 & $n s$ & .00 & 1.61 & $n s$ & .12 & 0.71 & $n s$ & .06 \\
\hline
\end{tabular}

Note: $N=13 ; \mathrm{ROI}=$ region of interest; $\mathrm{L}=$ left $\mathrm{R}=$ right. 
Table 2: Whole-brain analysis

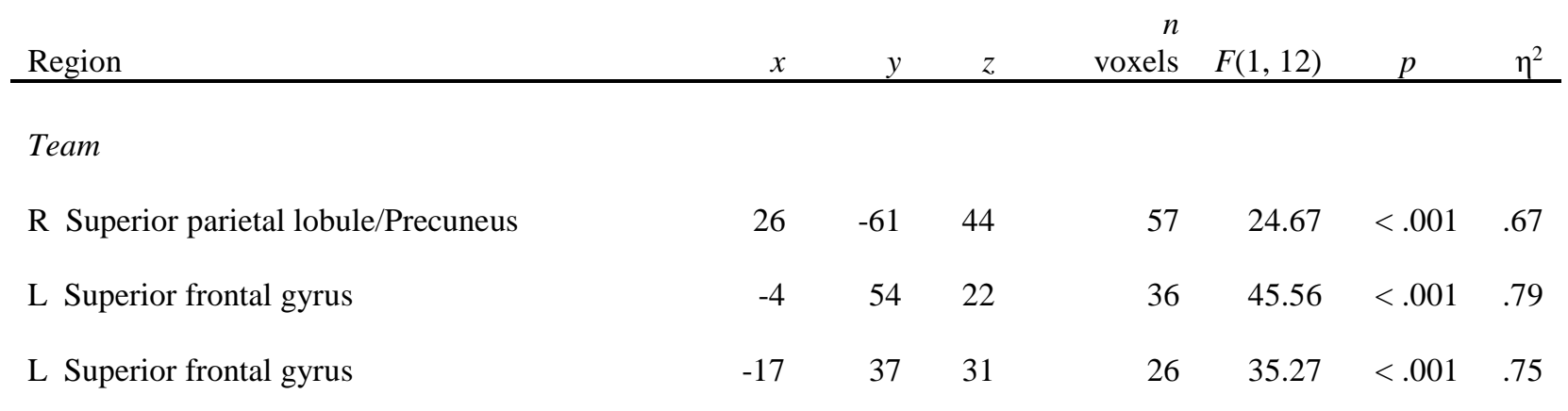

Situation

$\begin{array}{lrrrrrrr}\text { R Postcentral gyrus } & 37 & -27 & 57 & 165 & 27.88 & <.001 & .70 \\ \text { L Cerebellum } & -15 & -46 & -17 & 74 & 32.59 & <.001 & .73\end{array}$

Team $\times$ Situation

$\begin{array}{llllllllll}\text { L Inferior parietal lobule/Supramarginal gyrus } & -45 & -39 & 41 & 82 & 43.81 & <.001 & .79\end{array}$

$\begin{array}{lllllllll}\text { R Insula } & 45 & 3 & 15 & 70 & 34.44 & <.001 & .74\end{array}$

$\begin{array}{lllllllll}\mathrm{R} \text { Cingulate gyrus } & 10 & -30 & 35 & 42 & 21.83 & =.001 & .65\end{array}$

$\begin{array}{llllllll}\text { L Precuneus } & -6 & -55 & 47 & 39 & 34.37 & <.001 & .74\end{array}$

$\begin{array}{lllllllll}\text { L Cerebellum } & -12 & -39 & -40 & 30 & 41.82 & <.001 & .78\end{array}$

Note: $N=13 ; \mathrm{L}=$ left; $\mathrm{R}=$ right; $\mathrm{n}$ voxels = number of active, contiguous voxels in a cluster. 
1) The odds that the Team will WIN their first playoff game if their starting "Wide Receiver" PLAYS are

2) The odds that the Team will WIN their first playoff game if their starting "Wide Receiver" does NOT

PLAY are

3) The odds that the Team's starting "Wide Receiver" WILL PLAY are

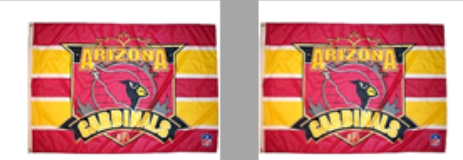

1) The odds that the Team will WIN their first playoff game if their starting "Wide Receiver" PLAYS are

2) The odds that the Team will WIN their first playoff game if their starting "Wide Receiver" does NOT

3) The odds that the Team's starting "Wide Receiver" WILL PLAY are

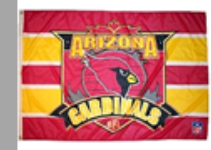

Arizona Cardinals
Please indicate what you believe to be the odds that the Arizona Cardinals will win their first playoff game.

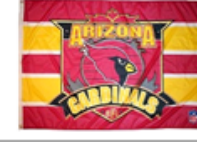


a

Deviation from

Objective Winning Odds in \%

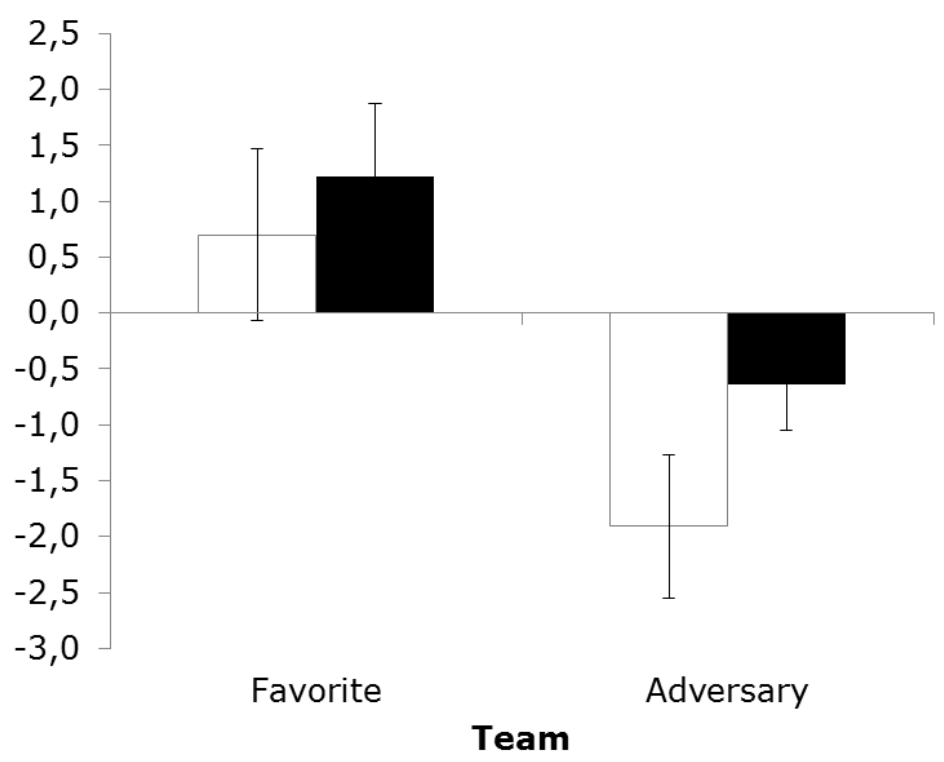

High Likelihood of Success b

Response Time in ms

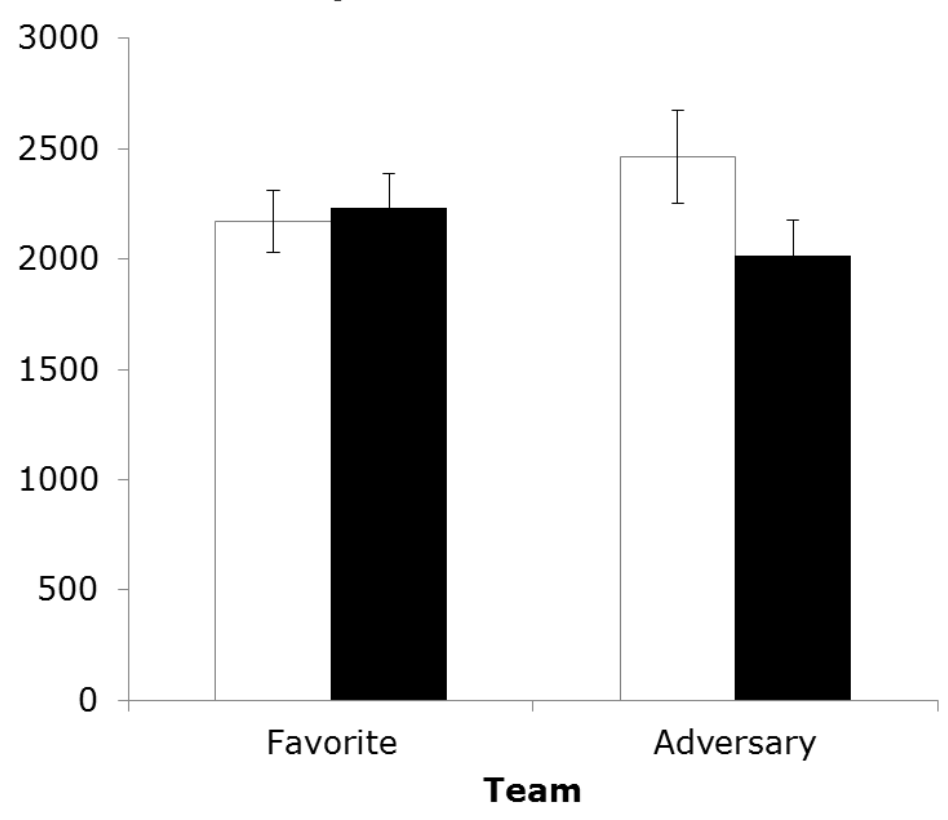

Low Likelihood of Success 
a

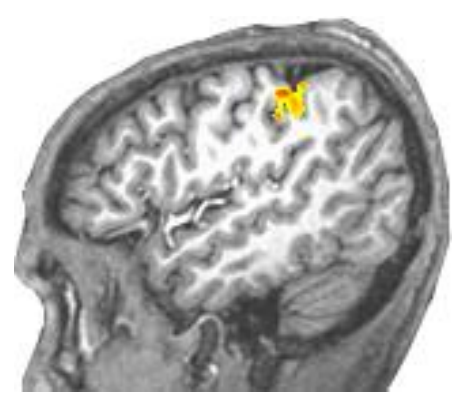

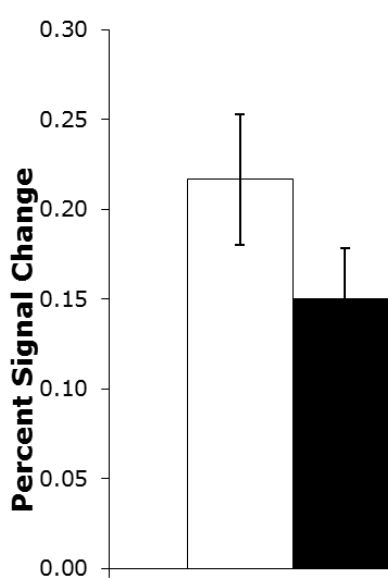

Favorite

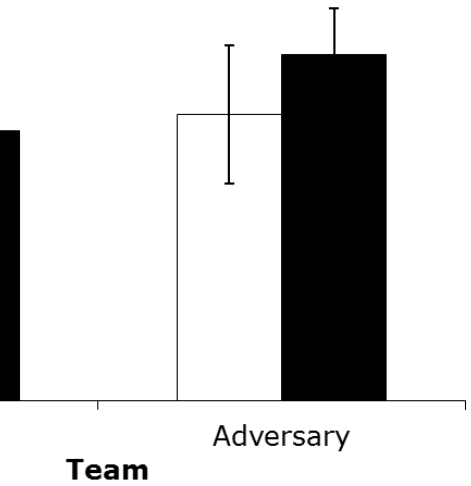

Right Insula

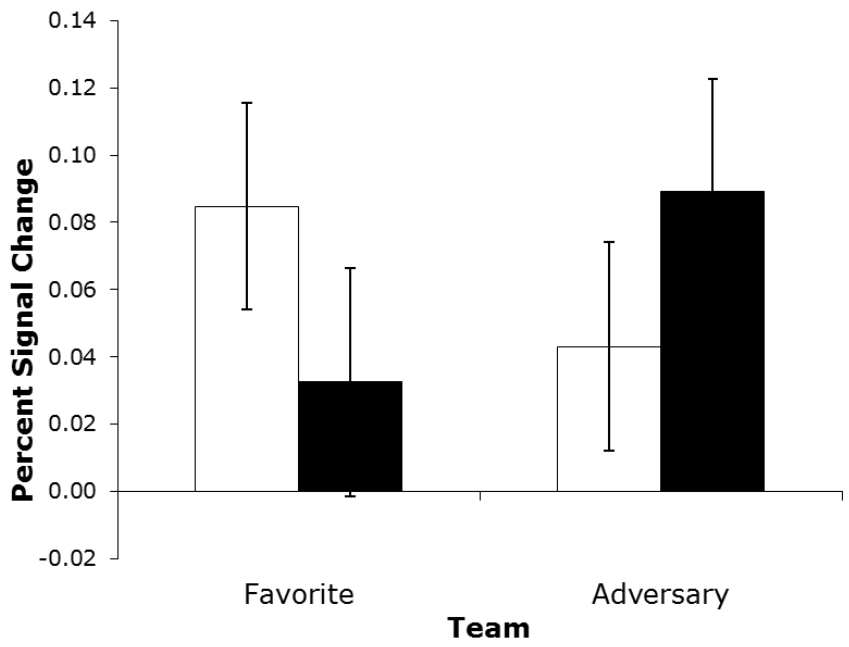

Right Cingulate Gyrus

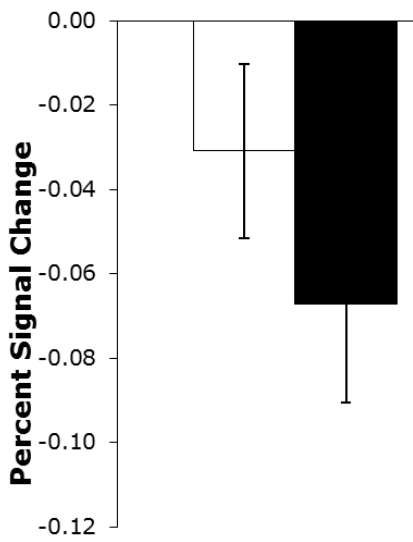

Favorite

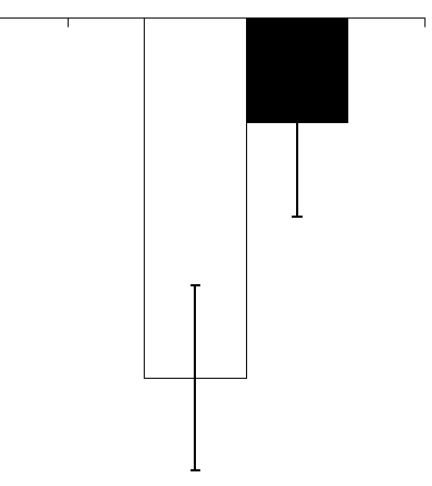

Adversary 


\section{Bilateral Precuneus}
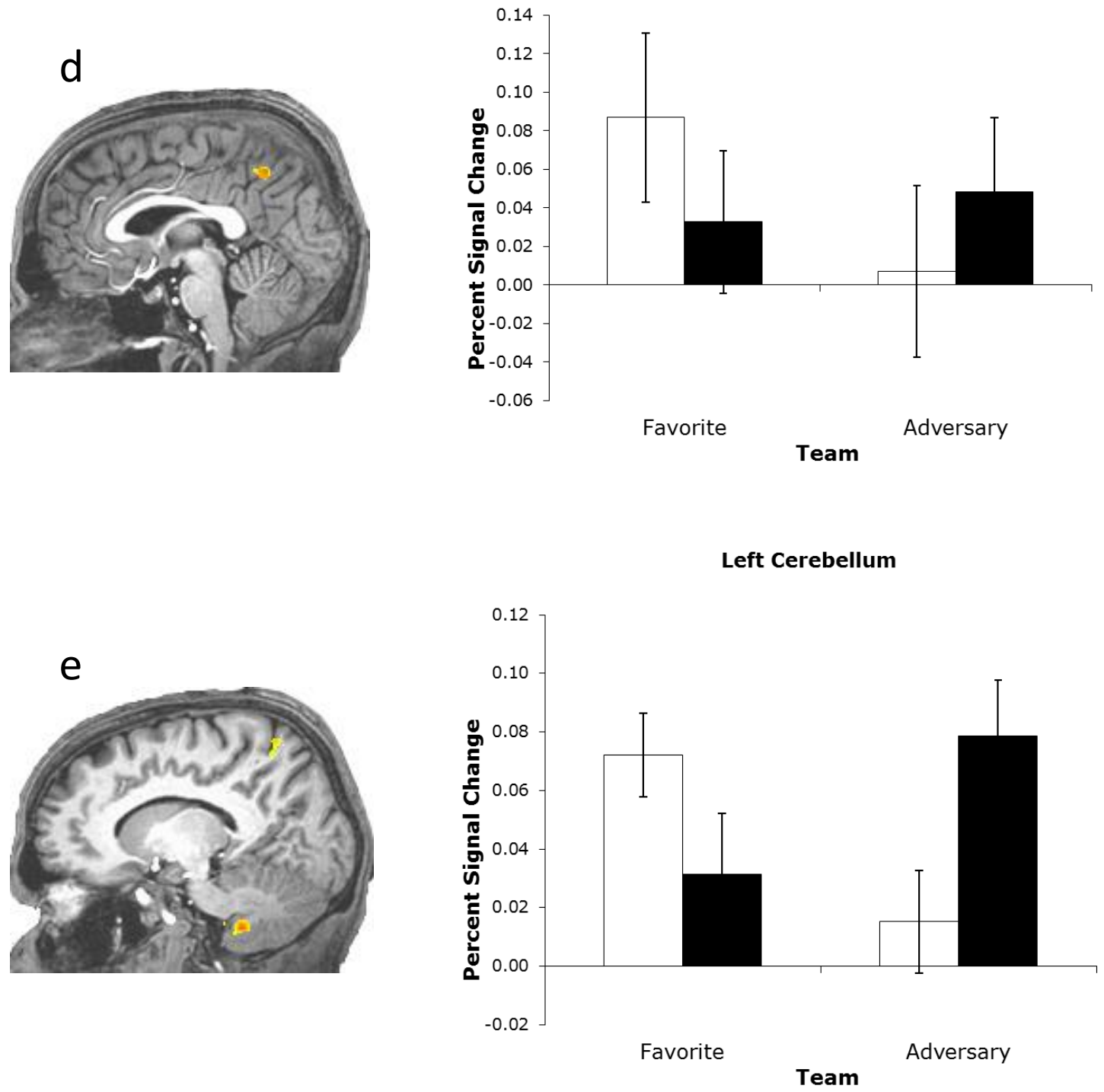

High Likelihood of Success

Low Likelihood of Success 